$\underline{\text { Supporting information file }}$

\title{
Measurements and Correlation of Isobaric \\ Vapor-Liquid Equilibria for the Binary Systems of \\ Cyclohexanol + Cyclohexyl Formate and \\ Cyclopentanol + Cyclopentyl Formate under \\ various pressures
}

\begin{abstract}
Tomáš Sommer, Martin Zapletal, Jiří Trejbal*
Department of Organic Technology, University of Chemistry and Technology Prague,

Technická 5, 16628 Prague 6, Czech Republic
\end{abstract}

*Corresponding Author

Email: Jiri.Trejbal@vscht.cz

Phone: +420220443689 
Table S1: Comparison of experimental vapor pressure of pure water obtained in this work and calculated vapor pressure by the Antoine parameters from the literature. ${ }^{1}$

\begin{tabular}{cccc}
$p^{\mathrm{s}} / \mathrm{kPa}$ & $T_{\exp } / \mathrm{K}$ & $T_{\text {cal }} / \mathrm{K}^{1}$ & $\sigma_{\mathrm{T}} / \%$ \\
97.71 & 372.35 & 372.15 & 0.054 \\
39.94 & 349.15 & 348.99 & 0.046 \\
25.02 & 338.25 & 338.14 & 0.033 \\
9.96 & 318.95 & 318.88 & 0.022 \\
\multicolumn{5}{l}{$\sigma_{\mathrm{T}}=100\left(T_{\exp }-T_{\text {cal }}\right) / T_{\exp }[\%]$} \\
\hline
\end{tabular}
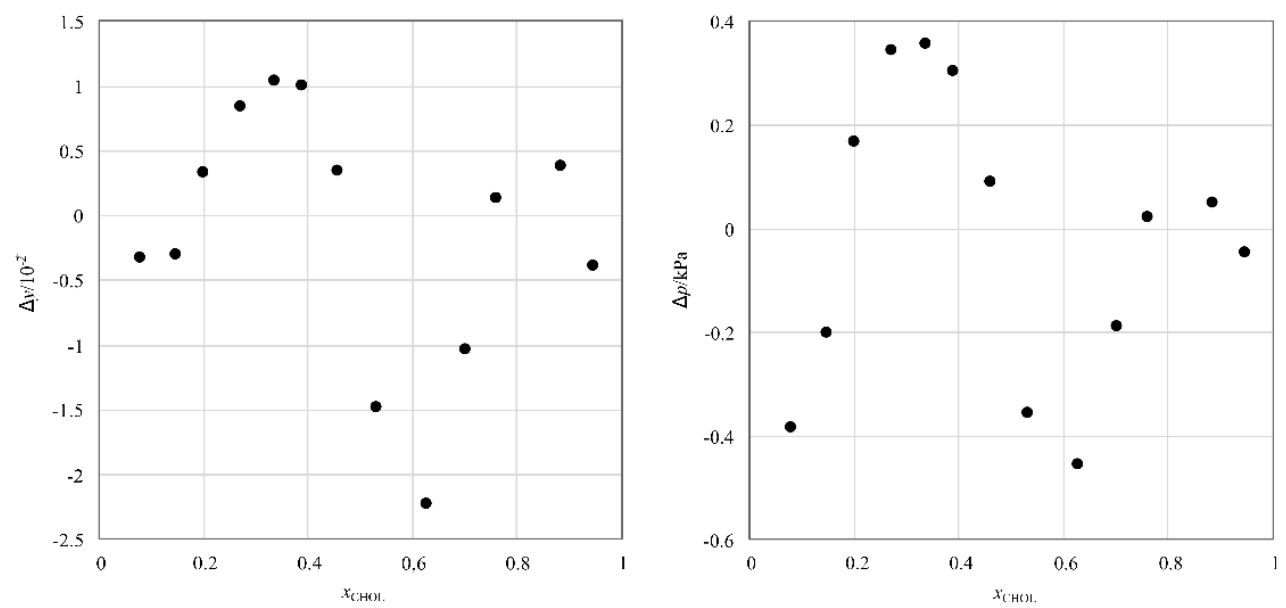

Figure S1: Distribution of residuals of vapor phase mole fraction and pressure under $10 \mathrm{kPa}$ for cyclohexanol + cyclohexyl formate binary system.
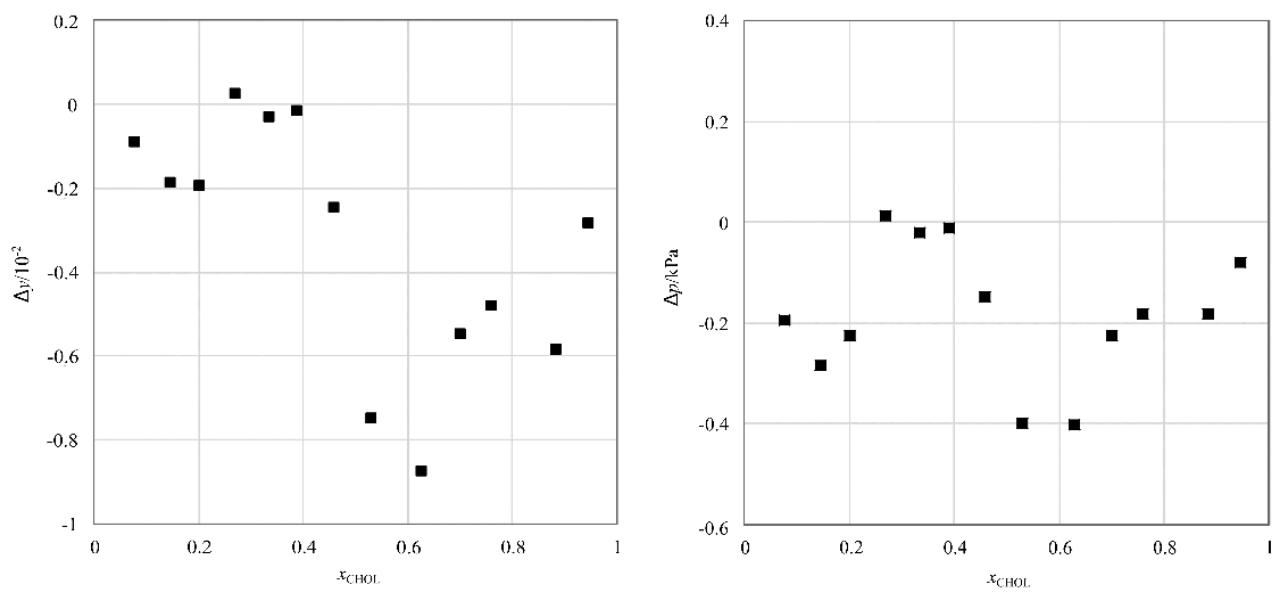

Figure S2: Distribution of residuals of vapor phase mole fraction and pressure under $25 \mathrm{kPa}$ for cyclohexanol + cyclohexyl formate binary system. 

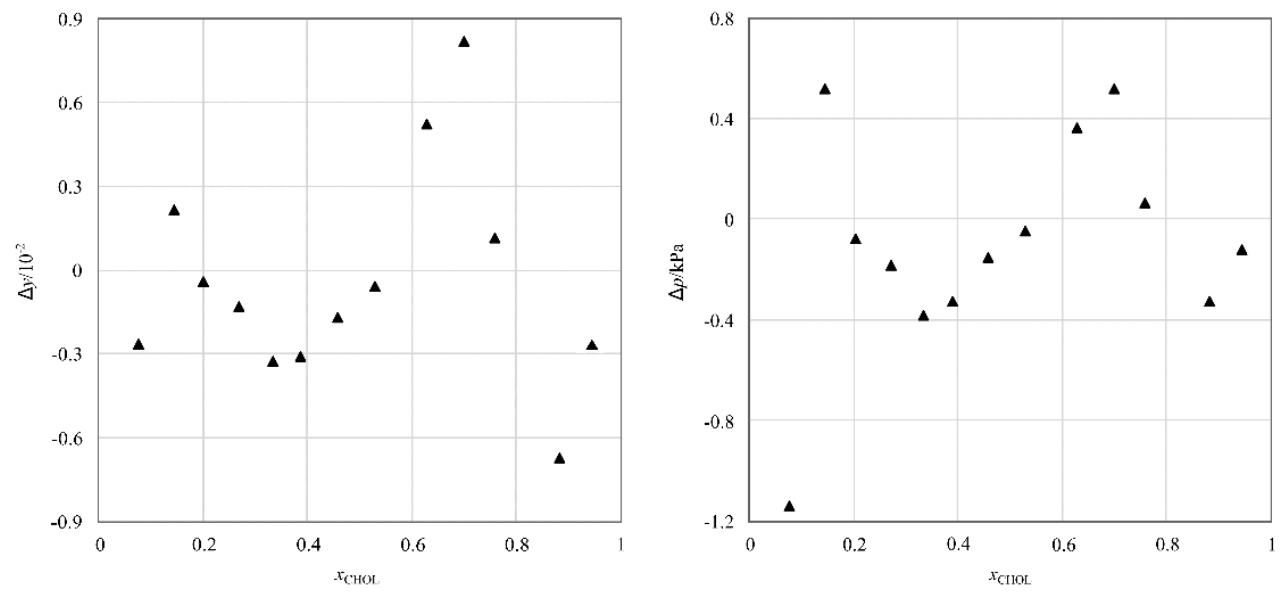

Figure S3: Distribution of residuals of vapor phase mole fraction and pressure under $40 \mathrm{kPa}$ for cyclohexanol + cyclohexyl formate binary system.
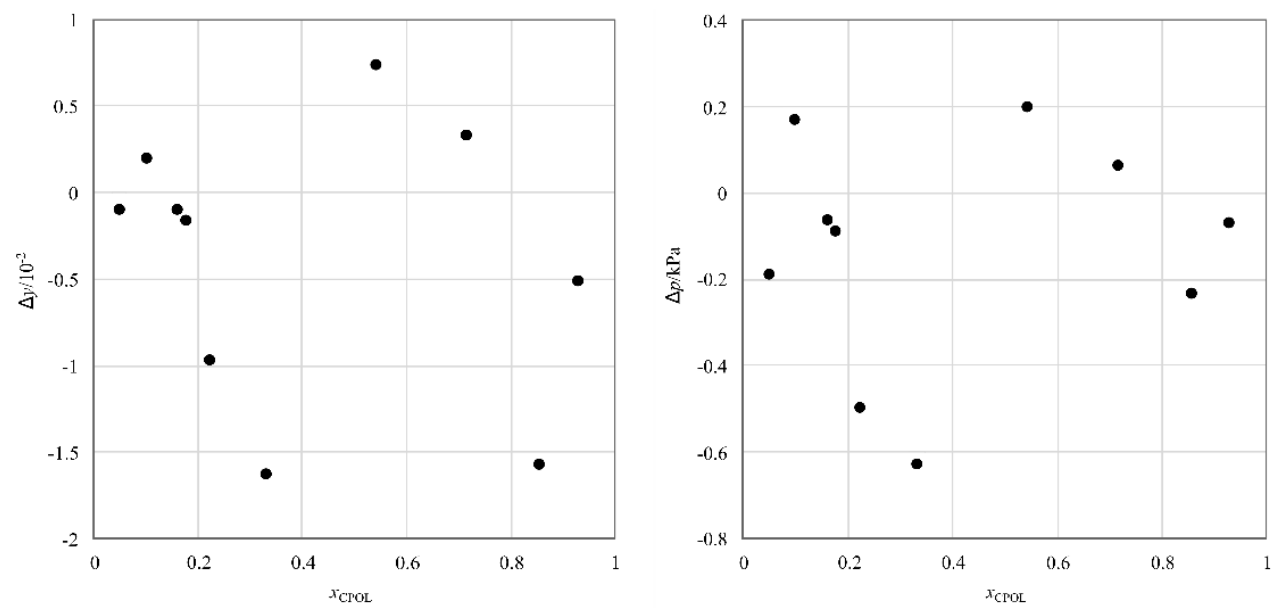

Figure S4: Distribution of residuals of vapor phase mole fraction and pressure under $10 \mathrm{kPa}$ for cyclopentanol + cyclopentyl formate binary system.
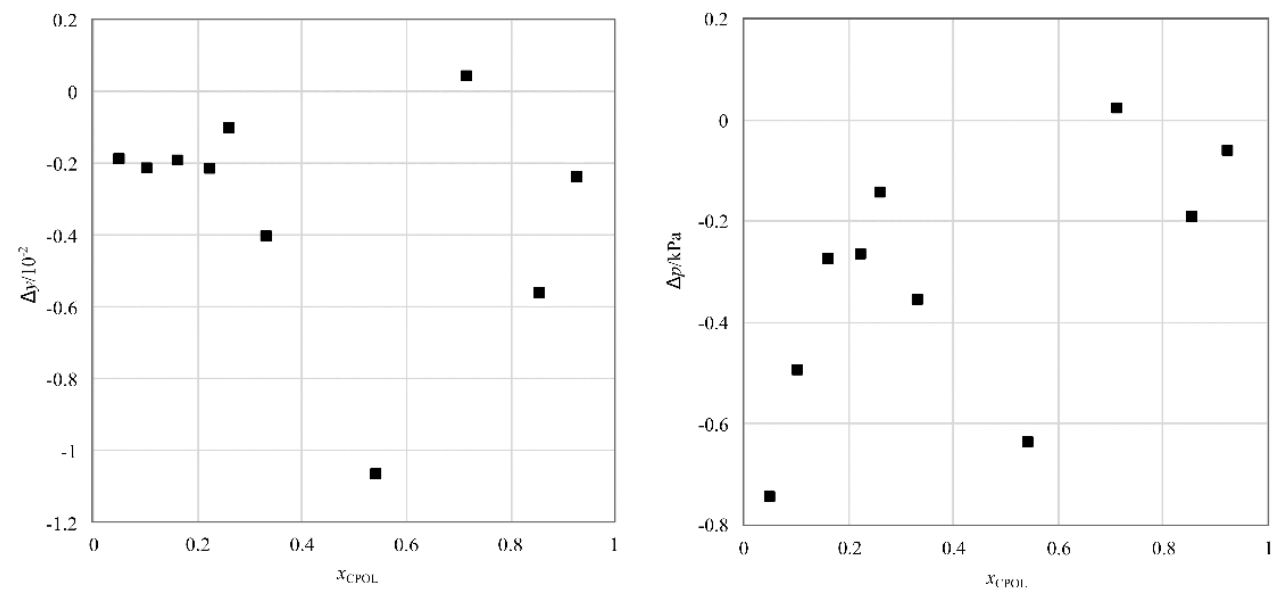

Figure S5: Distribution of residuals of vapor phase mole fraction and pressure under $25 \mathrm{kPa}$ for cyclopentanol + cyclopentyl formate binary system. 

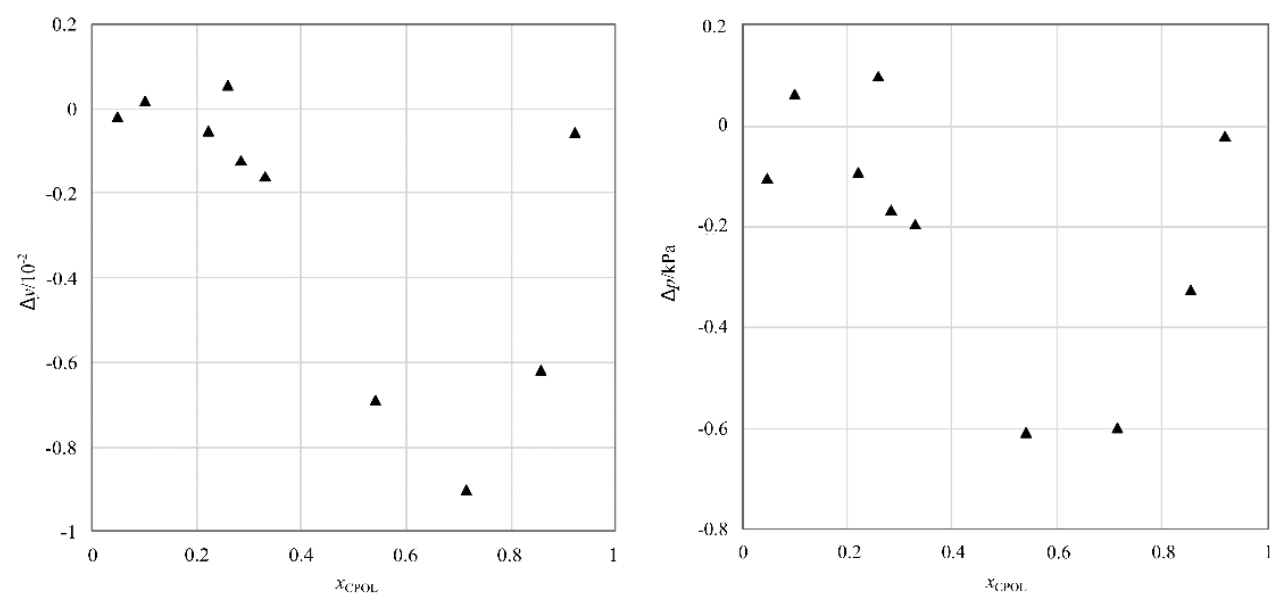

Figure S6: Distribution of residuals of vapor phase mole fraction and pressure under $40 \mathrm{kPa}$ for cyclopentanol + cyclopentyl formate binary system.

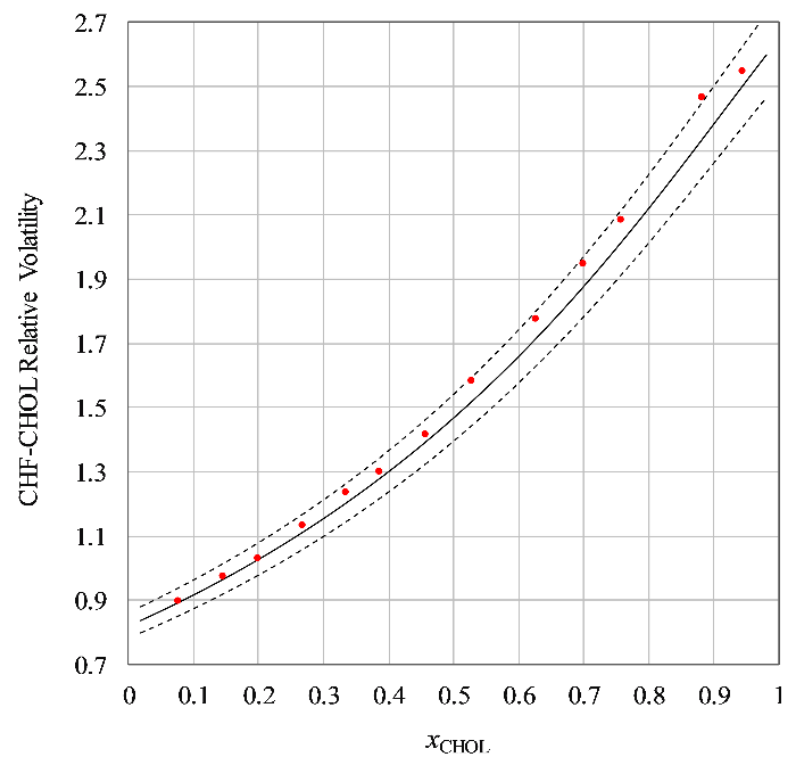

Figure S7: Relative volatilities of the cyclohexanol + cyclohexyl formate binary system under $10 \mathrm{kPa}$ : red $\bullet$, exp. data this work; black solid line, NRTL model this work, black dashed lines, $\pm 5 \%$ deviations from the calculated relative volatility. 


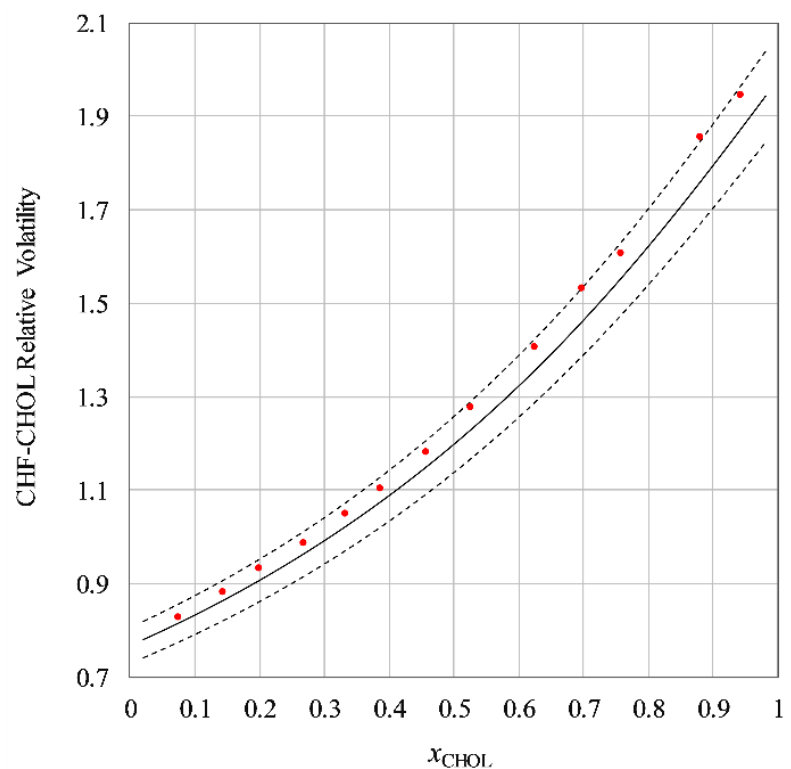

Figure S8: Relative volatilities of the cyclohexanol + cyclohexyl formate binary system under $25 \mathrm{kPa}$ : red $\bullet$, exp. data this work; black solid line, NRTL model this work, black dashed lines, $\pm 5 \%$ deviations from the calculated relative volatility.

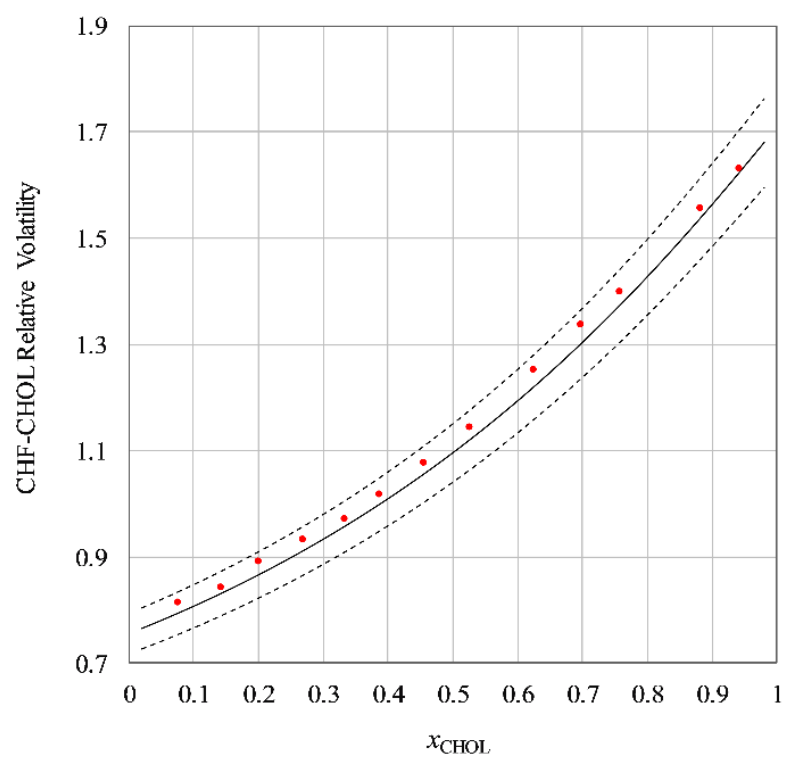

Figure S9: Relative volatilities of the cyclohexanol + cyclohexyl formate binary system under $40 \mathrm{kPa}$ : red $\bullet$, exp. data this work; black solid line, NRTL model this work, black dashed lines, $\pm 5 \%$ deviations from the calculated relative volatility. 


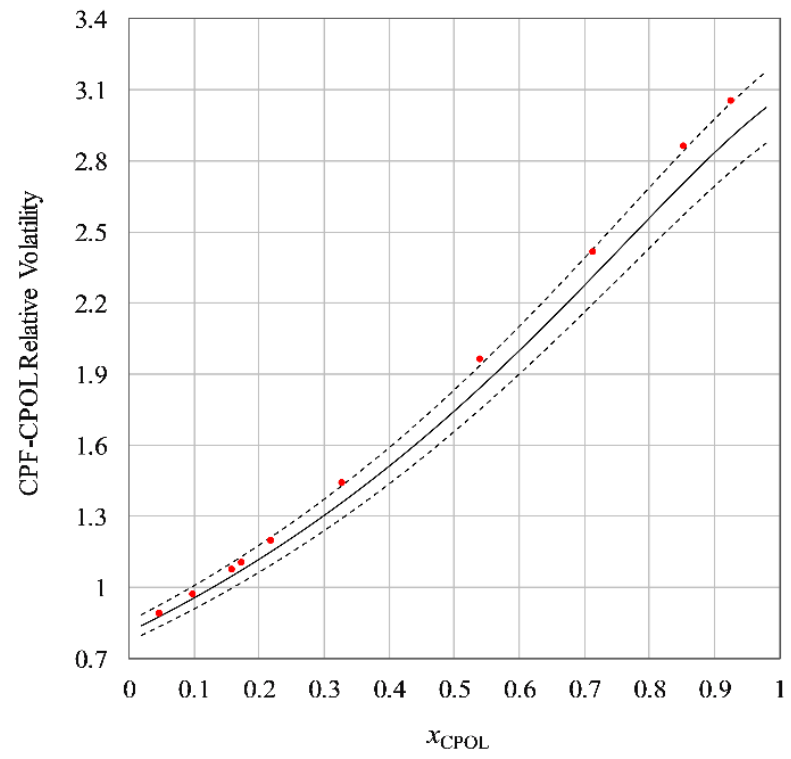

Figure S10: Relative volatilities of the cyclopentanol + cyclopentyl formate binary system under $10 \mathrm{kPa}$ : red $\bullet$, exp. data this work; black solid line, NRTL model this work, black dashed lines, $\pm 5 \%$ deviations from the calculated relative volatility.

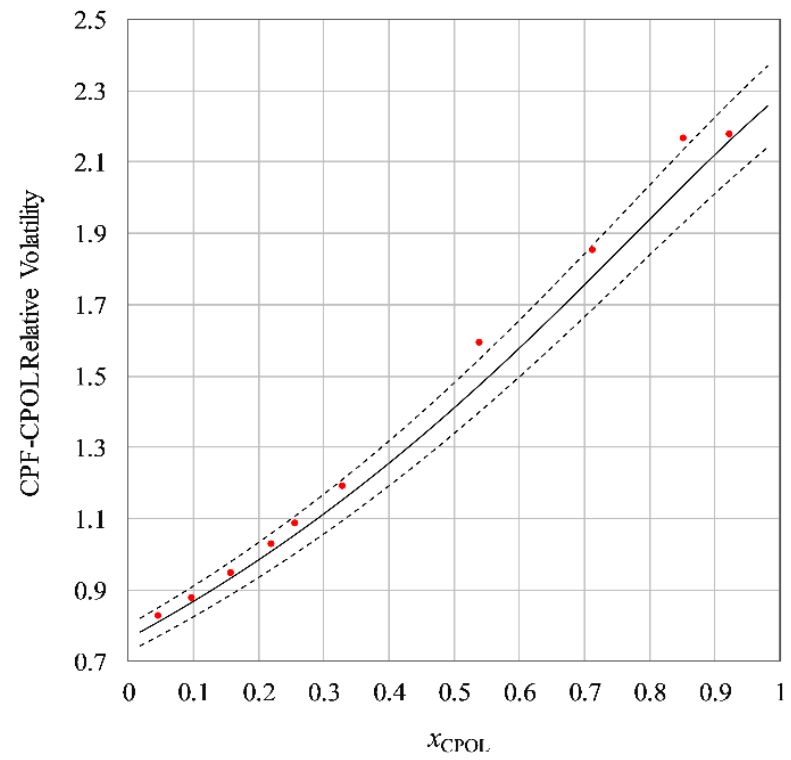

Figure S11: Relative volatilities of the cyclopentanol + cyclopentyl formate binary system under $25 \mathrm{kPa}$ : red $\bullet$, exp. data this work; black solid line, NRTL model this work, black dashed lines, $\pm 5 \%$ deviations from the calculated relative volatility. 


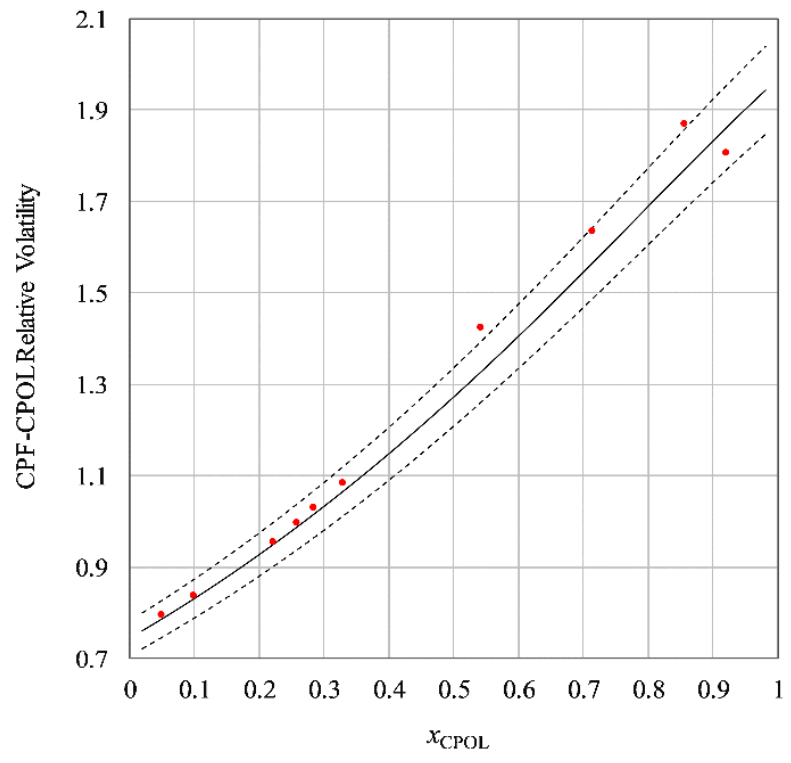

Figure S12: Relative volatilities of the cyclopentanol + cyclopentyl formate binary system under $40 \mathrm{kPa}$ : red $\bullet$, exp. data this work; black solid line, NRTL model this work, black dashed lines, $\pm 5 \%$ deviations from the calculated relative volatility.
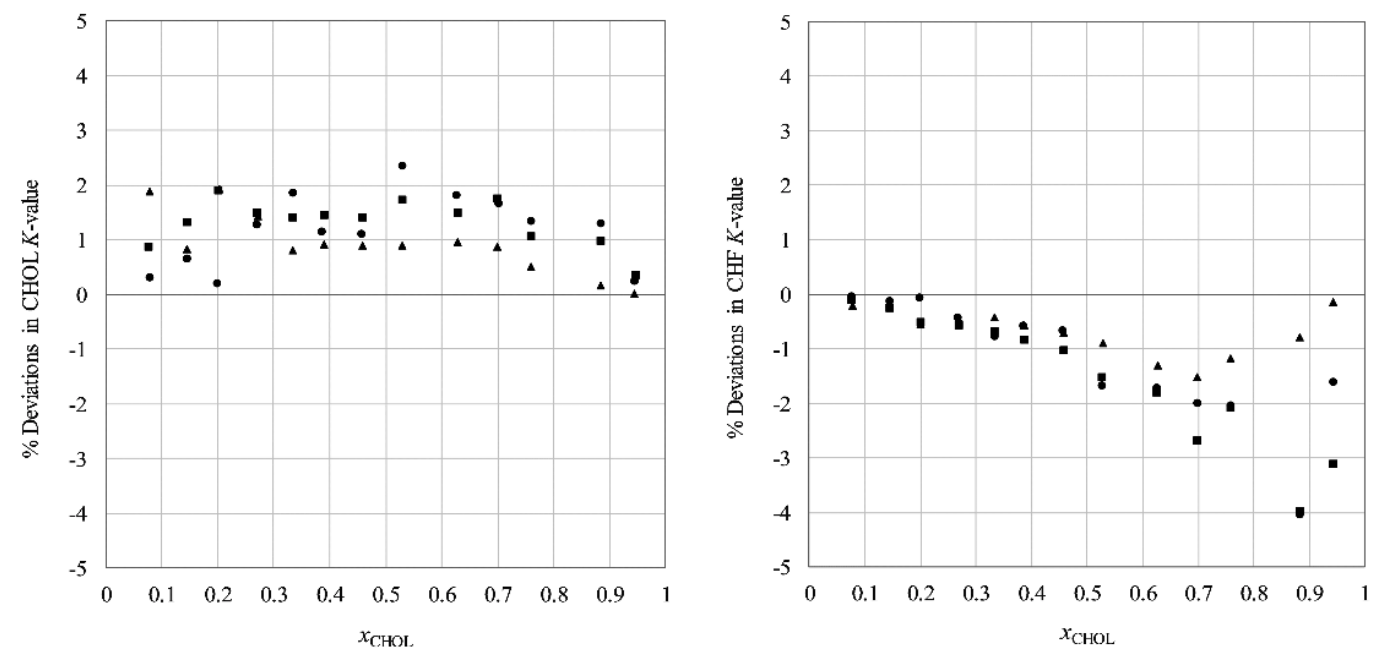

Figure S13: Percent deviations in $K$-values corresponding to cyclohexanol and cyclohexyl formate, respectively, between experimental and calculated data by NRTL model under various pressures: •, $10 \mathrm{kPa} ; \boldsymbol{\bullet}, 25 \mathrm{kPa} ; \boldsymbol{\Delta}, 40$ $\mathrm{kPa}$. 

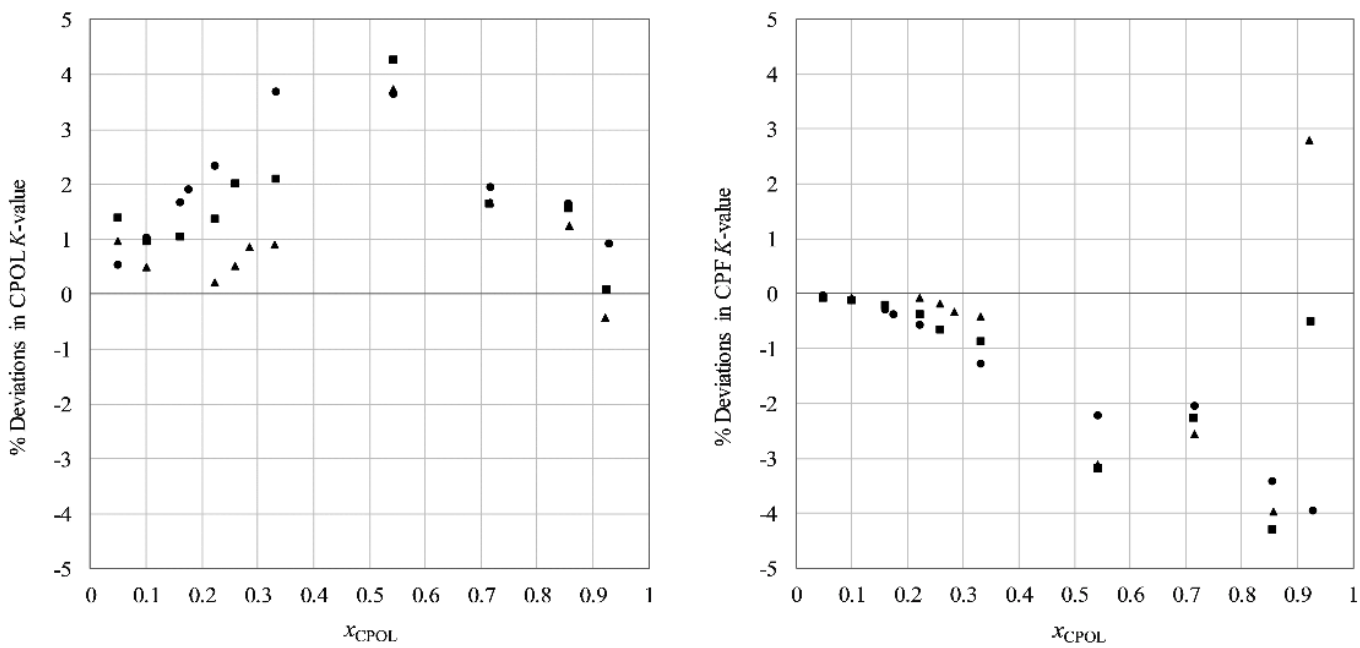

Figure S14: Percent deviations in $K$-values corresponding to cyclopentanol and cyclopentyl formate, respectively, between experimental and calculated data by NRTL model under various pressures: $\bullet, 10 \mathrm{kPa} ; \boldsymbol{\bullet}, 25 \mathrm{kPa} ; \boldsymbol{\Delta}, 40$ $\mathrm{kPa}$.

\section{REFERENCES}

(1) Design Institute for Physical Properties, Sponsored by AIChE. DIPPR Project 801 - Full Version. Design Institute for Physical Property Research/AIChE. Retrieved from https://app.knovel.com/hotlink/toc/id:kpDIPPRPF7/dippr-project-801-full/dippr-project-801-full (accessed October 2019). 